\title{
miR-186-5p targeting SIX1 inhibits cisplatin resistance in non-small-cell lung cancer cells (NSCLCs)
}

\author{
X. LIU, X. ZHOU, Y. CHEN, Y. HUANG, J. HE, H. LUO* \\ Department of Respiratory and Critical Care Medicine, The First Affiliated Hospital of the Army Medical University, Chongqing, China \\ *Correspondence: luohucy888@163.com
}

Received May 11, 2019 / Accepted September 4, 2019

\begin{abstract}
miR-186-5p has been reported to be implicated in tumorigenesis and paclitaxel resistance in non-small-cell lung cancer cells (NSCLCs). However, it remains undisclosed whether miR-186-5p takes a part in chemoresistance against diaminodichloroplatinum (cisplatin, DDP) in lung cancers, including NSCLC. Expression of miR-186-5p and sine oculis homeobox 1 (SIX1) was detected using RT-qPCR and western blot. In vitro, 50\% inhibitory concentration (IC50) of DDP and cell proliferation were measured by MTT assay. The rate of apoptosis and abilities of migration and invasion were evaluated with flow cytometry and Transwell assay. The target binding between miR-186-5p and SIX1 was predicted on Diana tools software and confirmed by dual-luciferase reporter assay and RNA immunoprecipitation. In vivo experiments, xenogeneic transplantation was conducted to monitor the tumor growth. Expression of miR-186-5p was downregulated in DDP resistant NSCLC tissues and cells (A549/DDP and H1299/DDP). Functionally, miR-186-5p overexpression could inhibit cell proliferation, migration and invasion, and promoted apoptosis rate in A549/DDP and H1299/DDP cells. Mechanically, SIX1 was identified as a downstream target for miR-186-5p and was highly expressed in A549/DDP and H1299/DDP cells. Similarly, SIX1 knockdown could also suppress DDP resistant NSCLC cell proliferation, migration and invasion, and promote apoptosis rate, which was reversed by miR-186-5p downregulation. Moreover, xenograft tumors induced by A549/DDP cells exerted cisplatin resistance, and miR-186-5p overexpression could inhibit tumor growth under DDP treatment. In conclusion, upregulation of miR-186-5p suppresses cisplatin resistance in DDP resistant NSCLC cells both in vitro and in vivo presumably by targeting SIX1.
\end{abstract}

Key words: miR-186-5p, SIX1, cisplatin resistance, NSCLC

Non-small-cell lung cancer (NSCLC) is one of the most lethal cancers worldwide and accounts for approximately $85 \%$ of all primary lung cancers [1]. NSCLC tumors are classified into three different subtypes: squamous cell carcinoma (SCC), adenocarcinoma (ADC) and large cell carcinoma (LCC). Chemotherapy is the major treatment approach for patients with NSCLC, and diaminodichloroplatinum (cisplatin, DDP) is one of the most effective and widely used chemotherapeutic drugs among cancers, including NSCLC $[2,3]$. Following the use of cisplatin chemotherapy, many changes have been associated with the multidrug-resistance phenotype of tumor cells. However, the clinical outcomes are disappointing, largely due to a poor response to cisplatin in these patients [4]. Therefore, it is urgently important to figure out the molecular mechanism of cisplatin resistance to further prevent NSCLC progression and recurrence.
MicroRNAs (miRNAs) are a group of endogenous small non-coding RNAs with approximately 22 nucleotides in length. Functionally, miRNAs are involved in diverse cellular processes, including proliferation, apoptosis, migration, etc. [5]. Besides, it is well recognized that miRNAs are important regulators of various pathophysiological processes in carcinogenesis [6], as well as drug resistance [7]. Recent studies have reported that miRNA (miR)-186-5p functions as tumor suppressor in various malignancies. In NSCLC, miR-186-5p has been shown to have lower expression and takes part in its tumorigenesis, development and prognosis [8-10]. Moreover, accumulating evidences have suggested a link between miR-186-5p and chemoresistance with several chemotherapeutic drugs, for instance paclitaxel $[11,12]$, methotrexate [13], platinum [14] and DDP [12, 15, 16]. In NSCLC, miR-186-5p is declared to regulate paclitaxel resistance of NSCLC cells both in vivo and in vitro [11]. 
However, its role in regulating cisplatin resistance has not been investigated in NSCLC.

Mechanically, miRNAs usually bind to 3' untranslated regions (3' UTR) of target genes, thus leading to their mRNA degradation or translational inhibition [17]. miR-186-5p exerts its biological functions through downregulating target genes as well [8-11]. In this study, we investigated the altered expression of miR-186-5p in DDP resistant and sensitive NSCLC tissues and cells. The role and molecular mechanism of miR-186-5p on cell proliferation, apoptosis, migration and invasion of DDP resistant NSCLC cells were evaluated in vitro, as well as on tumor growth in vivo.

\section{Materials and methods}

Acquirement of tissue samples. Before clinical sample acquirement, we had achieved the approval of Research Ethics Committee of the First Affiliated Hospital of the Army Medical University and the written informed consents from 50 NSCLC patients from 2014 to 2017. All patients received definitive chemotherapy with cisplatin after surgery. NSCLC tissue samples were obtained and divided into 2 groups: DDP resistant $(n=25)$ and DDP sensitive $(n=25)$. All tissue samples were immediately frozen in liquid nitrogen and then stored at $-80^{\circ} \mathrm{C}$. DDP resistant NSCLC was defined as tumor progression or recurrence within 6 months after the last DDP treatment, while those recurrence or progression more than 6 months were identified as DDP sensitive NSCLC.

Cells and cell culture. Human NSCLC cell lines A549 and H1299 were purchased from American Type Culture Collection (ATCC; Manassas, VA, USA). Cells were cultured in RPMI-1640 medium (Hyclone, Logan, UT, USA) containing $10 \%$ fetal bovine serum (FBS; Hyclone) and 1\% penicillin/ streptomycin (Invitrogen, Carlsbad, CA, USA) at $37^{\circ} \mathrm{C}$ with $5 \% \mathrm{CO}_{2}$.

Construction of DDP resistant NSCLC cells in vitro. DDP-resistant A549 and H1299 cells (A549/DDP and H1299/DDP) were developed with incubation in medium containing gradually increased concentrations of DDP (Sigma-Aldrich, St. Louis, MO, USA) from $5-30 \mu \mathrm{M}$ for 12 days. Finally, $5 \mu \mathrm{M}$ of DDP was additionally added into the medium to maintain the resistance phenotype of A549/DDP and H1299/DDP.

Cell transfection. miR-186-5p mimic, miR-186-5p inhibitor and siRNA against sine oculis homeobox 1 (si-SIX1) were purchased from Ribobio (Guangzhou, China), along with the negative controls. Cell transfection of oligonucleotides into A549/DDP and H1299/DDP cells was performed by Lipofectamine 2000 reagent (Invitrogen) according to the manufacturer's instructions. Cells were subsequently cultured for another $24 \mathrm{~h}$ prior for further study.

Lentivirus infection. Pre-miR-186-5p was amplified and cloned into pLV-EF1 $\alpha$-MCS-IRES-puro vector (Biosettia, San Diego, CA, USA). 293T cells were co-transfected with pLV-EF1a-MCS-IRES-puro-miR-186-5p/empty vector and
ViraPower Lentiviral Pack aging Mix (Thermo Fisher Scientific, Waltham, MA, USA) using Lipofectamine 2000 reagent (Invitrogen). After transfection for 3 days, the cell medium supernatant was collected as lentivirus particles carrying $\mathrm{miR}-186-5 \mathrm{p} / \mathrm{NC}$ (named as lenti-miR-186-5p/control). The construction of expression plasmid and the package of lentivirus particle were provided by Ribobio. A549/DDP and H1299/DDP cells were infected with lenti-miR-186-5p/ control, followed by screening with $2 \mu \mathrm{g} / \mathrm{ml}$ puromycin lasting for 14 days.

Total RNA isolation and real-time quantitative PCR (qPCR). Total RNA from tissue samples and cultured A549/DDP and H1299/DDP cells was extracted with Qiagen miRNeasy Mini kit (Qiagen, Hilden, Germany) following the manufacturer's protocol. The concentration and purity of total RNA was examined by NanoDrop 2000 spectrophotometer (Thermo Fisher Scientific). A reverse transcription kit (Abcam, Cambridge, UK) was used to synthesize the first strand of cDNAs. Then, amplification of cDNAs was performed by SYBR ${ }^{\oplus}$ Green Master Mix Kit (Qiagen) on an Applied Biosystems 7500 Real-Time PCR System (Thermo Fisher Scientific). The relative expression levels of miR-186-5p and SIX1 mRNA were calculated by $2^{-\triangle \Delta C T}$ methods with normalization to U6 small nuclear RNA (U6) and glyceraldehyde-3-phosphate dehydrogenase (GAPDH), respectively. All PCR reactions were performed in triplicate. Primers were listed as following: miR-186-5p: 5'-ACACTCCAGCTGGGCAGCAGCACACT-3' (forward) and 5'-CTCAACTGGTGTCGTGGA-3' (reverse); SIX1: 5'-AAGGAGAAGTCGAGGGGTGT-3' (forward) and 5'-TGCTTGTTGGAGGAGGAGTT-3' (reverse); GAPDH: 5'-GTCAACGGATTTGGTCTGTATT-3' (forward) and 5'-AGTCTTCTGGGTGGCAGTGAT-3' (reverse); U6: 5'-GCTTCGGCAGCACATATACTAAAAT-3' (forward) and 5'-CGCTTCACGAATTTGCGTGTCAT-3' (reverse).

Total protein extraction and western blot. Treated A549/DDP and H1299/DDP cells were extracted for total protein with RIPA lysis buffer (Beyotime, Shanghai, China) to measure SIX1 protein expression. Western blot was performed according to standard procedures, and $\beta$-actin on the same membrane was used as a loading control. The primary antibodies included SIX1 (sc-514441, 1:1000) and $\beta$-actin (sc-47778, 1:1000) and were purchased from Santa Cruz Biotech, CA, USA. The proteins were visualized using ECL procedure and Image J was used to analyze the gray intensity of the bands.

MTT assay. Cell proliferation of A549/DDP and H1299/ DDP cells was assessed by 3-(4,5)-dimethylthiazole-2-y1)-2, 5-biphenyl tetrazolium bromide (MTT) staining. Transfected cells were plated in 96-well plates (Corning, NY, USA) at a concentration of 5,000 cells per well and incubated with DDP. As for cisplatin resistance assay, transfected A549/DDP and H1299/DDP cells were treated with different concentrations of DDP $(5-30 \mu \mathrm{M})$ for $48 \mathrm{~h}$; as for cell proliferation ability detection, transfected A549/DDP and H1299/DDP 
cells were treated with $60 \mu \mathrm{M}$ of DDP for $0,24,48$, and $72 \mathrm{~h}$. Briefly, $20 \mu \mathrm{l}$ of MTT ( $5 \mathrm{mg} / \mathrm{ml}$; Sigma-Aldrich, Louis, MO, USA) was added to each well and the cells were incubated with MTT for another $4 \mathrm{~h}$. After adding $150 \mu \mathrm{l}$ of dimethyl sulfoxide (DMSO; Sigma-Aldrich), the spectrophotometric absorbance of each sample was measured at $450 \mathrm{~nm}$. The $50 \%$ inhibitory concentration (IC50) was calculated with GraphPad Prism 4.0 (GraphPad Software Inc, La Jolla, CA, USA) using the sigmoidal dose-response function. The experiments were conducted at least 3 times.

Transwell assay. For determination of abilities of migration and invasion, A549/DDP and H1299/DDP cells after transfection were evaluated with Transwell assay. Transfected cells were transferred in 24-well transwell chamber ( $8 \mu \mathrm{m}$ pores; Corning) with matrigel-free (for migration) or matrigel-coated (for invasion) (BD Biosciences). Transfected cells $\left(2 \times 10^{4} \mathrm{cells} / \mathrm{ml}\right)$ were re-suspended into $200 \mu \mathrm{l}$ of serumfree medium and plated in the upper chamber, and the lower chamber was filled with $500 \mu \mathrm{L}$ complete medium containing $10 \% \mathrm{FBS}$. After incubation at $37^{\circ} \mathrm{C}$ for $24 \mathrm{~h}$, the cells on the lower surface were stained with $0.1 \%$ crystal violet for $15 \mathrm{~min}$ at room temperature, followed by being photographed and counted under a light microscope.

Dual luciferase reporter assay and RNA immunoprecipitation (RIP). SIX1 3' UTR wild type and mutant type (SIX1 3' UTR-WT/MUT) was cloned into pGL4 vector (Promega, Madison, WI, USA). A549/DDP and H1299/DDP cells were co-transfected with SIX1 3' UTR-WT/MUT and miR-186-5p/NC mimic. All transfection procedures were performed by Lipofectamine 2000 (Invitrogen). $48 \mathrm{~h}$ posttransfection, the luciferase activity was measured using dualluciferase reporter system (Promega). The ratio of Firefly to Renilla luciferase activity was used as the relative luciferase activity. All operations were repeated 3 times.

Cell supernatant of A549/DDP and H1299/DDP after transfection of miR-186-5p/NC mimic was collected for RIP assay. Magna RIP ${ }^{\mathrm{m}}$ RNA-binding protein immunoprecipitation kit (Millipore, Bradford, MA, USA) was chosen to detect expression of SIX1 mRNA from the samples bound to the Ago2 or IgG antibody. All operation obeyed the standard instructions.

Flow cytometry. Apoptosis rate was analyzed by Annexin V-FITC/PI kit (Beyotime, Shanghai, China) on flow cytometry. After transfection, apoptotic cells were labelled with FITC-Annexin V and PI for $30 \mathrm{~min}$ in the dark and fluorescence was analyzed on CytoFLEX LX flow cytometer (Beckman-Counter Electronics, Jiangsu, China) using CytExpert software. Quadrants were positioned on Annexin V/PI plots to distinguish apoptotic cells (Annexin V+/PI-, Annexin $\mathrm{V}+\mathrm{PI}+)$. Apoptosis rate $=$ apoptotic cells $/$ total cells $\times 100 \%$

Xenograft mouse model. Four-week-old BALB/c nude mice were obtained from Model Animal Research Center of Nanjing University. The animal experiments were approved by The Institutional Review Board of the First Affiliated
Hospital of the Army Medical University and were taken in accordance with National Institutions of Health Guide for Care and Use of Laboratory Animals. Equal numbers $\left(1 \times 10^{6}\right)$ of A549/DDP cells stably infected with Lenti-miR186-5p or Lenti-miR-NC in $0.2 \mathrm{ml}$ of PBS were injected in subcutaneous area of nude mice (4 mice per group). One week later, xenograft experiments were divided into three groups: Lenti-control (Lenti-miR-NC+PBS), Lenti-miRNC (+DDP), Lenti-miR-186-5p (+DDP). DDP or PBS was intraperitoneally injected at a dose of $5 \mu \mathrm{g} / \mathrm{kg}$ every 7 days for five times. The tumors were measured with a caliper once 7 days, and tumor volume was calculated using the formula: $V\left(\mathrm{~mm}^{3}\right)=1 / 2 a b^{2}$ ( $a$ is the longest tumor axis and $b$ is the shortest tumor axis). The mice were euthanized on day 35 after xenograft and the weight of tumors was evaluated with electronic balance. Immediately, the xenograft tumors were snap-frozen in liquid nitrogen and stored in $-80^{\circ} \mathrm{C}$ for further isolation of total RNA and protein.

Statistical analyses. Data given were the means \pm standard deviation. Statistical significance was determined by two-tailed Student's $t$ test and one-way analysis of variance using GraphPad Prism 4.0 (GraphPad Software Inc). A p-value $<0.05$ was considered as significant difference.

\section{Results}

Expression of miR-186-5p was downregulated in DDP resistant NSCLC tissues and cells. In order to explore the contribution of miR-186-5p on cisplatin resistance in NSCLC, altered expression of miR-186-5p was investigated. As shown in Figure 1A, miR-186-5p expression was universally downregulated in DDP resistant NSCLC tumor tissues $(n=25)$ comparing with DDP sensitive tissues $(n=25)$. We developed DDP resistant NSCLC cells in A549 and H1299 (named as A549/DDP and H1299/DDP) for further functional experiments. Moreover, the cultivated A549/ DDP and H1299/DDP cells were identified by analyzing 50\% inhibitory concentration (IC50) of DDP. As a result, IC50 of DDP in cultivated A549/DDP and H1299/DDP cells was more than $60 \mu \mathrm{M}$, whereas about $30 \mu \mathrm{M}$ in the parental cells (Figures $1 \mathrm{~B}$ and $1 \mathrm{C}$ ), which suggested a successful construction for DDP resistant NSCLC cells in vitro. Expression levels of miR-186-5p were lower in A549/DDP and H1299/DDP cells than in the parental cells (Figure 1D). These findings showed the downregulation of miR-186-5p in DDP resistant NSCLC.

Overexpression of miR-186-5p inhibited proliferation, migration and invasion, and promoted cell apoptosis in DDP resistant NSCLC cells in vitro. We confirmed that miR-186-5p was downregulated in DDP resistant NSCLC, then miR-186-5p was highly expressed in A549/DDP and H1299/DDP cells by transfection with miR-186-5p mimic (Figure 2A). Loss-of-function experiments were conducted to figure out the role of miR-186-5p overexpression. IC50 of DDP in A549/DDP and H1299/DDP cells was dramati- 
A

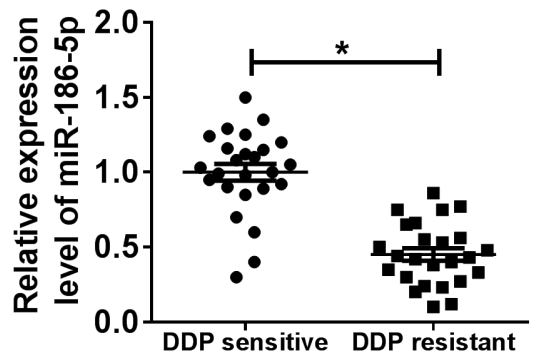

C

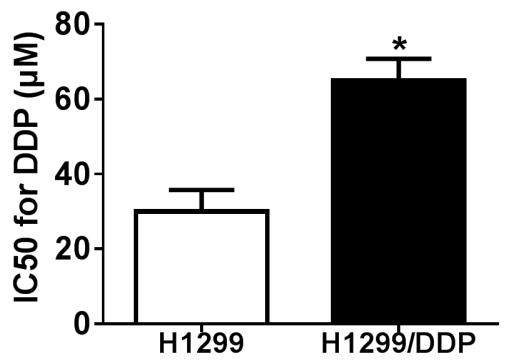

B

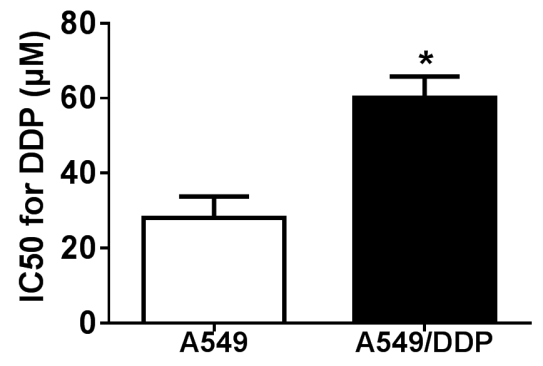

D

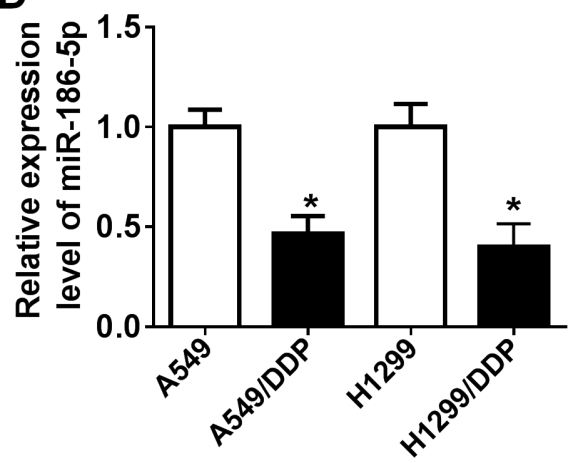

Figure 1. miRNA (miR)-186-5p was downregulated in DDP-resistant NSCLC tissues and cells. A) Levels of miR-186-5p were detected by RT-qPCR in DDP resistant $(n=25)$ and sensitive $(n=25)$ NSCLC tissues. B and C) IC50 of DDP was measured with cell viability assay. D) Levels of miR-186-5p were detected by RT-qPCR in A549/DDP and H1299/DDP cells, normalized to the parental cells. All experiments were carried out in triplicate, and ${ }^{\star}$ p $<0.05$.

cally decreased when miR-186-5p was overexpressed (Figure 2B). According to MTT staining, ectopic expression of miR-186-5p significantly inhibited cell proliferation ability in A549/DDP and H1299/DDP cells under $60 \mu \mathrm{M}$ of DDP treatment (Figures 2C and 2D). Abilities of cell migration and invasion were impaired in miR-186-5p-overexpressed A549/DDP and H1299/DDP cells, as evidenced by Transwell assays (Figures $2 \mathrm{E}$ and $2 \mathrm{~F}$ ). On the contrary, apoptotic cells were greatly induced when miR-186-5p overexpressed (Figure 2G). These results indicated upregulation of miR-186-5p inhibited proliferation, migration and invasion, and promoted cell apoptosis in DDP resistant NSCLC cells in vitro.

SIX1 was upregulated and was targeted by miR-186-5p in DDP resistant NSCLC cells. In this study, we observed 4 potential binding sites between hsa-miR-186-5p and SIX1 ENSG00000126778 by computational predictions on Diana tools. As shown in Figure 3A, the potential binding site on SIX1 3' UTR 2322-2337 was further validated. Then, the target sequence on SIX1 wild type (SIX1-WT) was cloned into pGL4 vector, as well as the mutant type (SIX1-MUT). Dual-luciferase reporter assay was used to validate the target binding of miR-186-5p and SIX1. A549/DDP and H1299/ DDP cells were co-transfected with SIX1 3' UTR-WT/MUT and $\mathrm{miR}-186-5 \mathrm{p} / \mathrm{NC}$ mimic (miR-186-5p/NC). Relative luciferase activity of SIX1 3' UTR-WT was remarkably reduced in the presence of miR-186-5p (Figures 3B and 3C); however, there was no difference in SIX1 3' UTR-MUT groups. RIP assay further identified this binding in A549/DDP and H1299/DDP cells. After transfection with miR-186-5p, levels of SIX1 mRNA from Ago2 immunoprecipitation were largely enriched (Figure 3D). Expression of SIX1 in DDP resistant NSCLC cells was uncovered either. Levels of SIX1 protein were higher in A549/DDP and H1299/DDP cells than parental cells (Figure 3E). Subsequently, the regulatory effect of miR-186-5p mimic and inhibitor was verified in A549/ DDP and H1299/DDP cells using RT-qPCR analyzing levels of miR-186-5p (Figures $2 \mathrm{~A}$ and $3 \mathrm{~F}$ ). Next, the regulatory effect of miR-186-5p on SIX1 expression was detected, and SIX1 expression on protein level was reduced when transfected with miR-186-5p but increased when transfected with miR-186-5p inhibitor (in-miR-186-5p) (Figure 3G). These data illustrated that miR-186-5p negatively regulated SIX1 through target binding and SIX1 was upregulated in DDP resistant NSCLC cells.

Knockdown of SIX1 suppressed proliferation, migration and invasion, and promoted cell apoptosis in DDP resistant NSCLC cells depending on miR-186-5p upregulation. We wondered whether miR-186-5p exerted suppressive effect on cisplatin resistance in NSCLC through inhibiting its target SIX1 expression. Thus, rescue experiments were performed. A549/DDP and H1299/DDP cells were transfected with si-SIX1/NC or co-transfected with si-SIX1 and in-miR-186-5p/NC. Expression levels of SIX1 were measured 

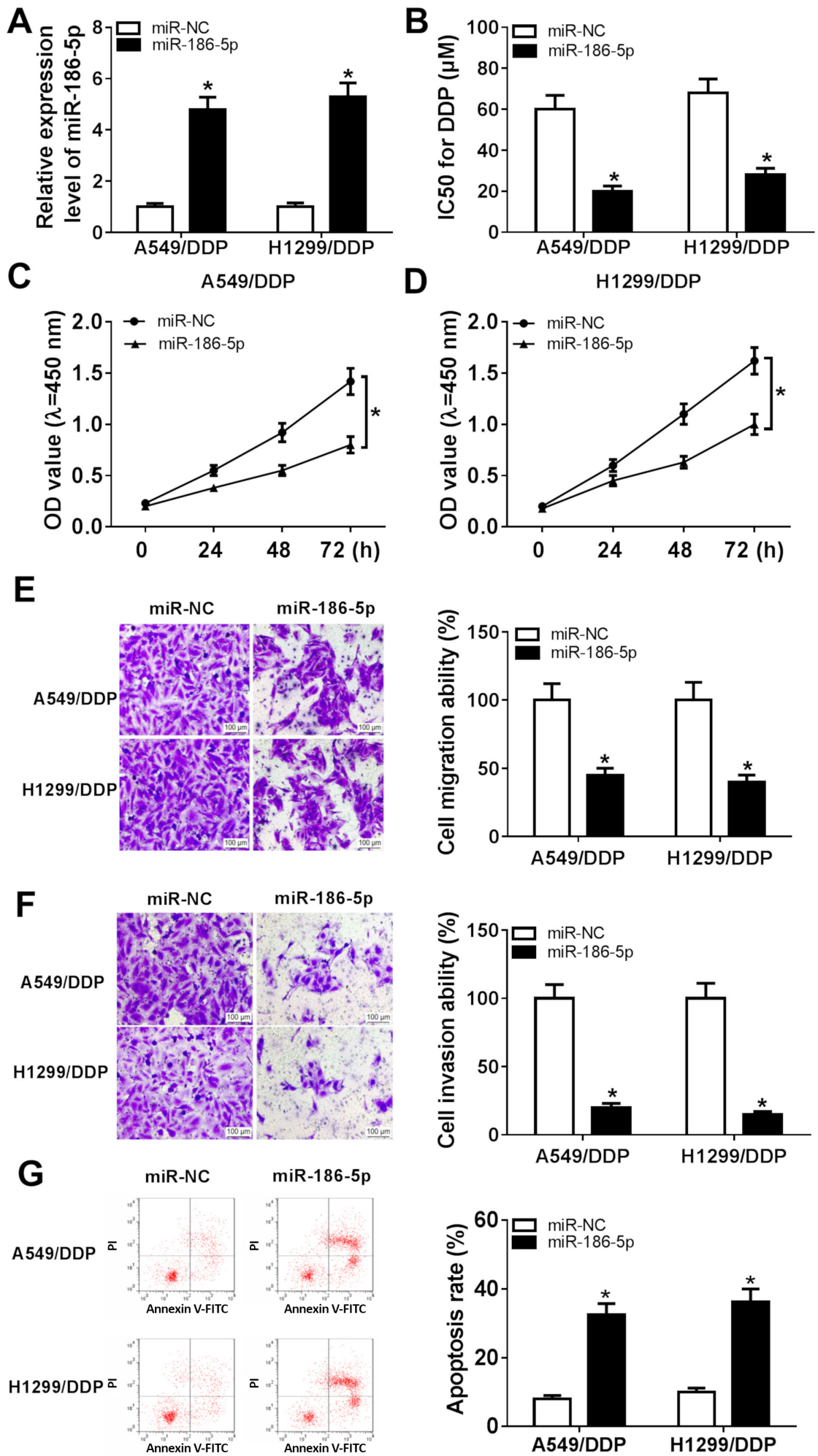

Figure 2. Effect of miR-186-5p overexpression on DDP resistant NSCLC cells in vitro. A549/DDP and H1299/DDP cells were transfected with miR-1865p mimic (miR-186-5p) or miR-NC mimic (miR-NC). A) Levels of miR-186-5p were detected with RT-qPCR after transfection. B) IC50 of DDP was measured with cell viability assay (with MTT) after transfection. C and D) Cell viability was measured in transfected A549/DDP and H1299/DDP cells after $60 \mu \mathrm{M}$ DDP treatment for $0,24,48$ and $72 \mathrm{~h}$. E and F) Ability of cell migration and invasion was examined with Transwell assay after transfection. G) Apoptosis was measured with apoptosis rate using flow cytometry. The percentage of cells in quadrants of Annexin V+/PI- and Annexin V+/PI+ was statistically recorded. All experiments were carried out in triplicate, and ${ }^{\star} \mathrm{p}<0.05$ versus miR-NC. 
A

\section{hsa-miR-186-5p \\ SIX1 3'UTR-WT \\ SIX1 3'UTR-MUT}

B

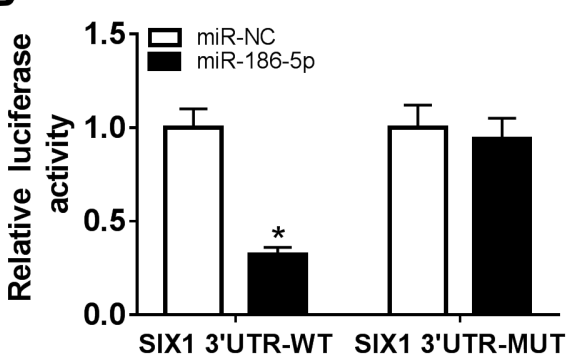

D

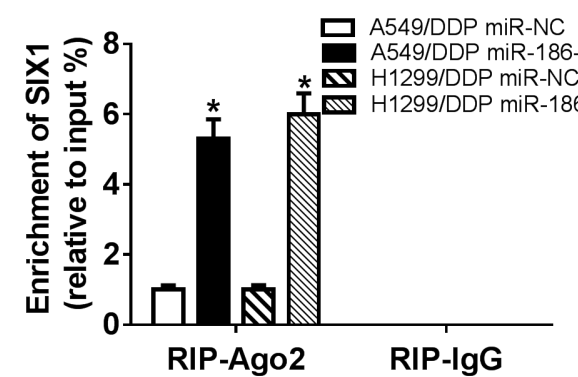

$\mathbf{F}$

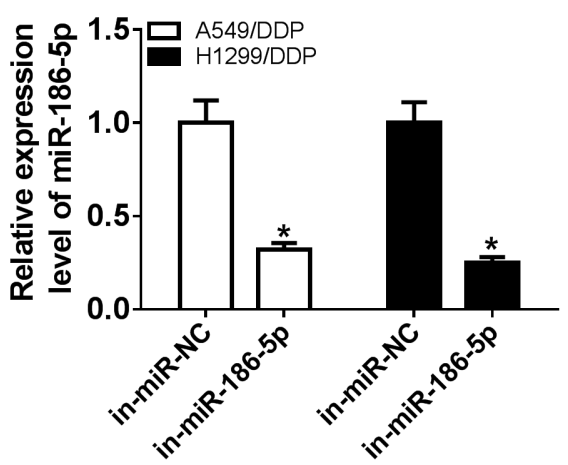

3' UCGgGUUUUCCUCUUAAGAAAC 5' 5' ...AAUACAAUCUAUAACUUUCUUUG... 3' 5 '...AAUACAAUCUAUAACUAAGAAAC... 3'

C H1299/DDP
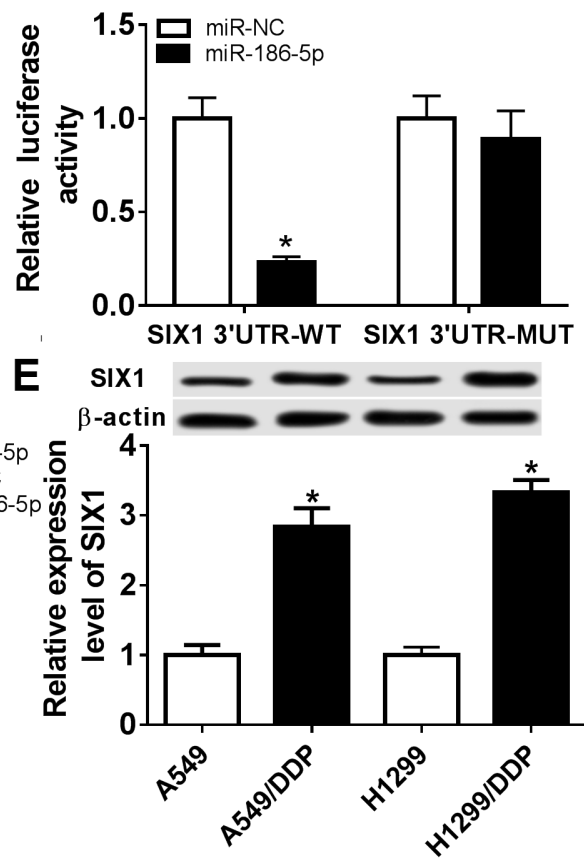

G
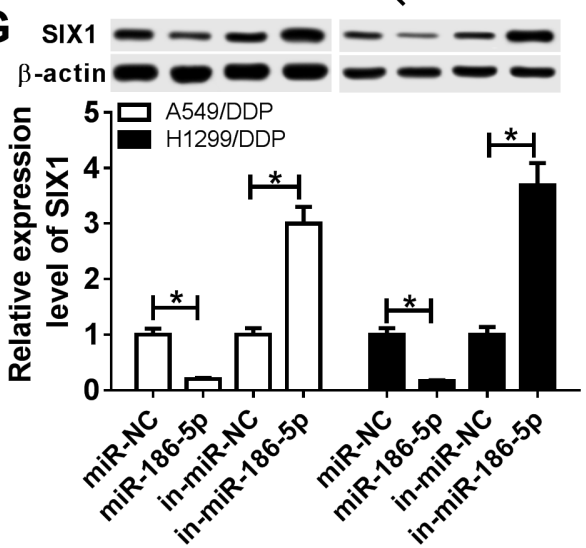

Figure 3. miR-186-5p negatively regulated sine oculis homeobox 1 (SIX1) expression by target binding. A) The potential binding site of miR-186-5p on SIX 3' UTR was shown. (B-D) A549/DDP and H1299/DDP cells were transfected with miR-186-5p or miR-NC. B and C) Relative luciferase of pGL4SIX 3' UTR wild type and mutant after transfection. D) Expression levels of SIX1 mRNA in RNA immunoprecipitation from Ago2 after transfection. E) Levels of SIX1 protein were measured by western blot in A549/DDP and H1299/DDP cells, compared with the parental cells. F) Levels of SIX1 mRNA were measured by RT-qPCR in A549/DDP and H1299/DDP cells when transfected with miR-miR-186-5p/NC inhibitor (in-miR-miR-186-5p/NC). G) The regulatory effect of miR-186-5p on SIX1 expression was detected using western blot. Levels of SIX1 protein were measured in A549/DDP and H1299/DDP cells when transfected with miR-miR-186-5p/NC or in-miR-miR-186-5p/NC. All experiments were carried out in triplicate, and ${ }^{\star} \mathrm{p}<0.05$ versus miR-NC or in-miR-NC.

after transfection; SIX1 levels were declined by transfection of si-SIX1 alone and improved when transfected with si-SIX1 together with in-miR-186-5p (Figure 4A). IC50 of DDP in A549/DDP and H1299/DDP cells was decreased with SIX1 knockdown, and then rescued when co-expression of SIX1 and miR-186-5p were concurrently inhibited (Figure 4B). SIX1 downregulation alone could inhibit cell proliferation ability in A549/DDP and H1299/DDP cells under $60 \mu \mathrm{M}$ of DDP treatment, which was abolished by low expression of miR-186-5p (Figures 4C and 4D). Abilities of cell migration 


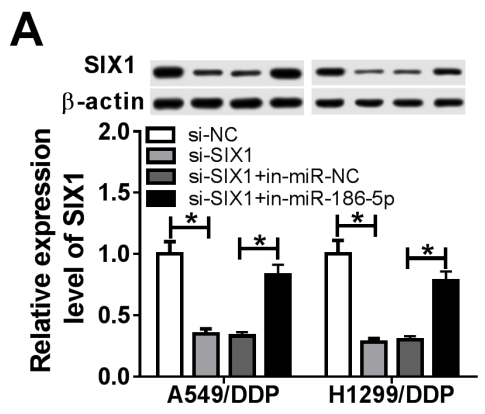

B

C

A549/DDP
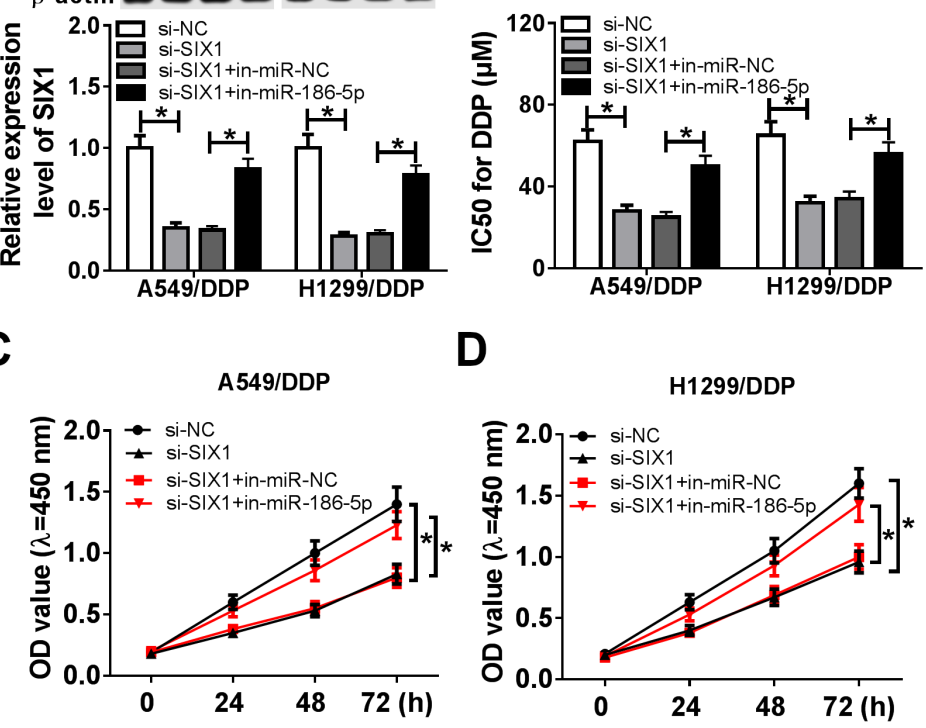

H1299/DDP

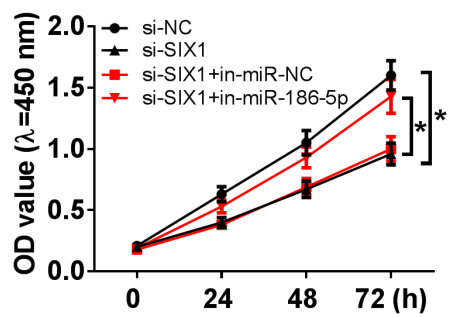

E

F
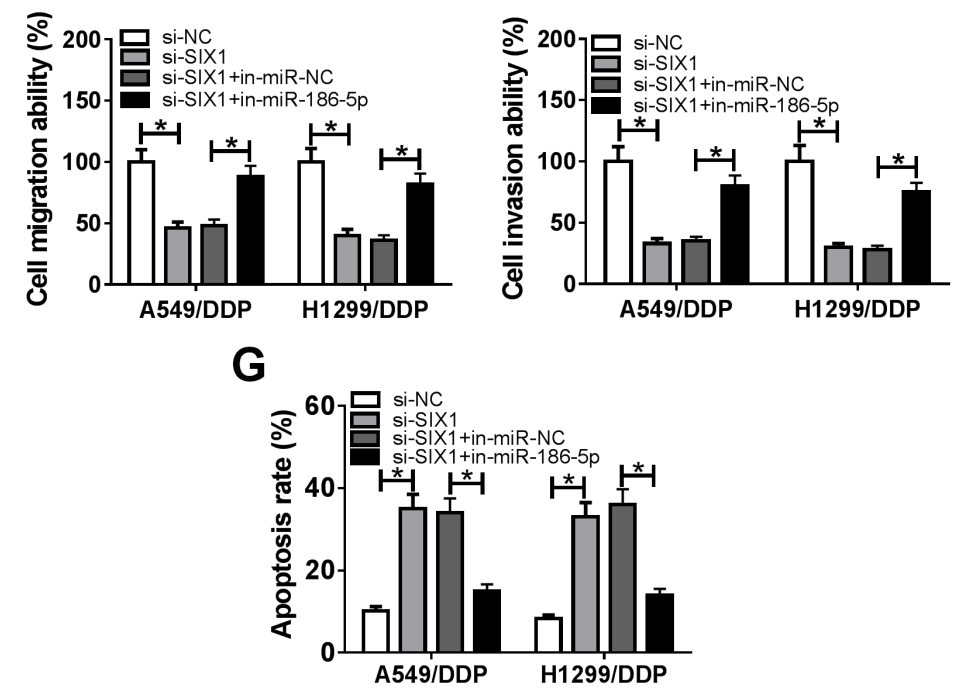

Figure 4. Knockdown of SIX1 suppressed DDP resistant NSCLC cell proliferation, migration and invasion depending on miR-186-5p upregulation. A549/DDP and H1299/DDP cells were transfected with siRNA SIX1/NC (si-SIX1/NC) or co-transfected with si-SIX1 and in-miR-186-5p/NC. A) Expression levels of SIX1 were measured by western blot after transfection. B) IC50 of DDP was measured with cell viability assay (with MTT) after transfection. C and D) Cell viability was measured after transfection followed with DDP treatment for 0, 24, 48 and $72 \mathrm{~h}$. E and F) Ability of cell migration and invasion was examined with Transwell assay after transfection. G) Apoptosis rate was measured by flow cytometry. All experiments were carried out in triplicate, and ${ }^{*} \mathrm{p}<0.05$ versus control cells transfected with si-NC or si-SIX1 and in-miR-NC.

and invasion were higher when transfected with si-SIX1, and this effect was reversed when co-transfected with si-SIX1 and in-miR-186-5p (Figures 4E and 4F). Inversely, apoptosis rate was greatly elevated when SIX1 was downregulated, which was partially reversed by simultaneously reduced miR-186-5p expression (Figure 4G). These results indicated that the effects of SIX1 knockdown in DDP resistant NSCLC cells in vitro were abated by miR-186-5p downregulation.
Overexpression of miR-186-5p suppressed xenograft NSCLC tumor growth with DDP treatment. In present study, we concluded that miR-186-5p sensitized the DDP resistant NSCLC cells to DDP in vitro. Then, we wondered whether miR-186-5p overexpression could suppress cisplatin resistance in vivo. A549/DDP cells stably expressing miR-186-5p by lentivirus infection were subsequently xenogeneic transplanted into nude mice. As shown in Figure 5, xenograft 
experiments were divided into three groups: Lenti-control (Lenti-miR-NC+PBS), Lenti-miR-NC (+DDP), Lenti-miR186-5p (+DDP). A549/DDP cells-induced xenograft tumor growth was significantly inhibited by enforced expression of miR-186-5p comparing to Lenti-miR-NC group (Figures 5A and $5 \mathrm{~B}$ ); while, there was no difference between miR-NC tumors with and without DDP treatment. Furthermore, we dug out the expression of miR-186-5p and SIX1 in xenograft tumors. As expected, miR-186-5p was highly upregulated and SIX1 was extremely downregulated in Lenti-miR-186-5p group (Figures 5C and 5D). These data showed NSCLC xenograft tumors were retarded by miR-186-5p under DDP treatment, implying the suppressive role of miR-186-5p on cisplatin resistance in NSCLC in vivo.

\section{Discussion}

Drug resistance still remains a major obstacle to the efficacy of chemotherapy, even though great advances have been achieved in adjuvant therapies [18]. Cancer chemotherapy is limited by the development of drug resistance and the adverse effects of anti-tumor drugs [19]. Besides, $70-80 \%$ of patients are diagnosed at advanced stages due to the limitation of early diagnostic techniques and the lack of early specific clinical manifestations [20]. Numerous studies have demonstrated that miRNAs play important roles in mediating DDP sensitivity of NSCLC cells [21]. On one hand, dysregulation of some miRNAs contributes to the acquisition of chemoresistance in NSCLC. For example, the inhibition of miR-155 could enhance the sensitivity of A549 cells to DDP treatment by modulation of cellular apoptosis and DNA damage through an Apaf-1-mediated pathway [22]. Wang et al. [23] indicated that miR-224 targeting p21 promoted the chemoresistance of human lung adenocarcinoma cells to DDP via regulating G1/S transition and apoptosis. On the other hand, altered expression of many miRNAs suppresses chemoresistance in NSCLC. For instance, garcinol sensitized NSCLC cells to erlotinib and cisplatin by upregulating epithelial-to-mesenchymal transition (EMT)-modulating miRNAs such as miR-200c and let-7c [24]. Sun et al. [25] highlighted increased miR-202 expression could expand apoptosis signaling induced by DDP in NCI-H441 and A549 NSCLC cells through targeting KRas and inhibiting Ras/MAPK pathway. In our study, miR-186-5p was identified as tumor suppressor in NSCLC and miR-186-5p upregulation inhibited cell proliferation,

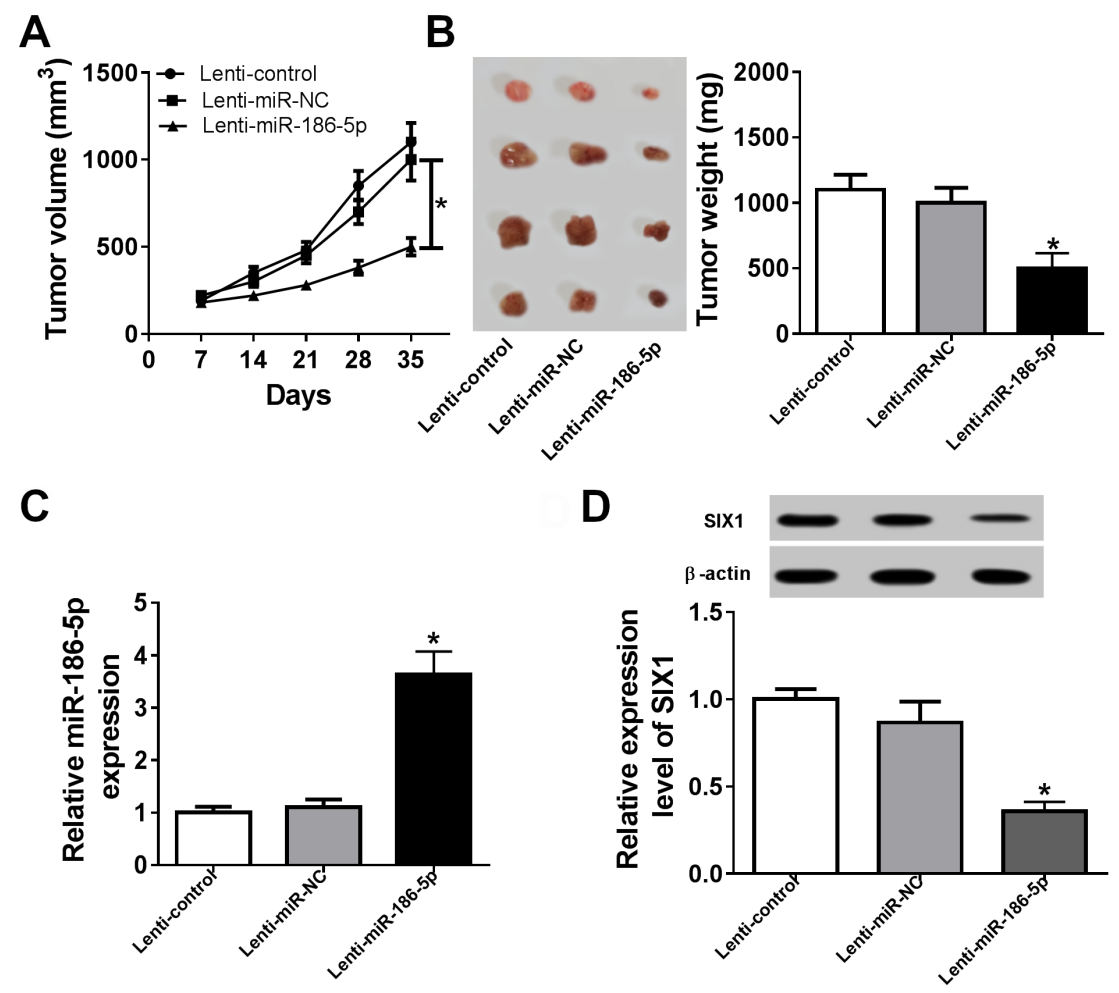

Figure 5. Effect of miR-186-5p overexpression on NSCLC tumor growth under DDP treatment. A549/DDP and H1299/DDP cells were infected with lentivirus particles carrying miR-186-5p/NC (Lenti-miR-186-5p/NC), followed by xenograft. After 7 days, xenograft experiments were divided into three groups: Lenti-control (Lenti-miR-NC+PBS), Lenti-miR-NC (+DDP), Lenti-miR-186-5p (+DDP). A) Tumor size was measured per 7 days and the growth curve was drawn. B) The representative images of tumors were presented and tumor weight was recorded on day 35 . C and D) Expression levels of miR-186-5p and SIX1 were detected by RT-qPCR and western blot, respectively. All experiments were carried out in triplicate, and ${ }^{\star} \mathrm{p}<0.05$ versus Lenti-miR-NC. 
migration and invasion, and promoted cell apoptosis rate in DDP resistant A549 and H1299 cells by downregulating its target SIX1 expression. Taken these data together, the molecular mechanism of cisplatin resistance may include regulation of miRNAs to decrease the cell proliferation and metastasis and to increase apoptosis.

miR-186-5p plays universal role in NSCLC tumorigenesis. Cai et al. [10] firstly claimed a close correlation between miR-186-5p expression and NSCLC patient survival. In that study, enforced overexpression of miR-186-5p in A549 and SK-MES-1 NSCLC cells inhibited the proliferation by inducing G1/S checkpoint arrest, and silencing its expression played the opposite effect. Then, overexpression of miR-186-5p was reported to inhibit invasion activity of NSCLC cells toward the SCID mice lung in research from $\mathrm{Li}$ et al. [26]. Moreover, a markedly decelerated proliferation of the tumor cells was caused by miR-186-5p upregulation [ 9 , $27,28]$. The mechanisms underlying the anti-tumor role of miR-186-5p were recently uncovered in A549 and HCC827 cells through targeting Rho-associated protein kinase 1 [28], mitogen-activated protein kinase kinase kinase 2 [27] and Yin Yang 1 [9]. In this study, we focused on the role of this miRNA on DDP-resistant NSCLC cells. As a result, ectopic expression of miR-186-5p resulted in the inhibition of IC50 values of DDP, cell proliferation, migration and invasion, and enhancement of apoptosis rate in A549/DDP and H1299/ DDP cells, as well as xenograft tumor growth. Notably, consistent downregulation of miR-186-5p was recognized in NSCLC tissues and cells, we further declared the lower expression level of miR-186 in DDP resistant NSCLC tissues and cells.

miR-186-5p was involved in the occurrence of chemotherapeutic drug-resistance in NSCLC. It was observed that downregulated miR-186-5p occurred in NSCLC patients who were paclitaxel resistant, and this downregulation was associated with poor survival [11]. In NSCLC, there was no published data indicating the involvement between cisplatin resistance and miR-186-5p expression, even though miR-186-3p was early uncovered to be downregulated in the anti-cancer effects of curcumin in A549/DDP cells [19]. Here, we firstly developed the DDP resistant NSCLC cells in A549 and H1299 cells, namely A549/DDP and H1299/DDP. Expression of miR-186-5p was downregulated in DDP resistant NSCLC patients and A549/DDP and H1299/DDP cells. Overexpression of miR-186-5p distinctively attenuated DDP resistant phenotypes in A549/DDP and H1299/DDP cells, including IC50 value of DDP, proliferation, apoptosis, migration and invasion. A549/DDP cells-induced xenograft tumor growth was significantly inhibited by enforced expression of miR-186-5p; while, there was no difference between miR-NC tumors with and without DDP treatment. SIX1 knockdown may underlay the suppressive role of miR-186-5p on cisplatin resistance in NSCLC in vitro and in vivo.

SIX1 belongs to the sine oculis homeobox family, which is a homologue of the Drosophila sine oculis in vertebrates [29].
SIX family genes play an important role in organogenesis and disease [30]. Among them, SIX1 is the most heavily implicated SIX protein in cancers [31]. Over past decades, SIX1 has been related to "hallmarks of cancer", such as maintained cell proliferation, activated migration and invasion, resisted cell death, and etc. In lung cancer, upregulated SIX1 and Notch2 were investigated to be associated with invasive lung ADC, a mainly subtype of NSCLC, by inducing EMT and nuclear atypia, thus promoting advanced malignant phenotypes [32]. Roles of miRNAs in SIX1-mediated functions had gained much attention in several cancers including NSCLC [33]. Moreover, SIX1 was closely correlated with drug resistance and poor prognosis in some human cancers, such as breast cancer [34] and ovarian cancer [35, 36]. However, to the best of our knowledge, the contribution of SIX1 to cisplatin resistance in many other cancers remains unclear, including lung cancer. In this present study, we figured out the influence of SIX1 in miR-186-5p-induced DDP sensitivity in NSCLC. As a result, SIX1 downregulation by siRNA could reduce IC50 values of DDP in A549/DDP and H1299/ DDP cells through inhibiting cell proliferation, migration and invasion and facilitating apoptosis. Furthermore, this effect was reversed by knockdown of miR-186-5p. Therefore, we concluded that miR-186-5p and SIX1 could be a new therapeutic target for DDP resistant NSCLC, even though the role and mechanism of SIX1 underlying cisplatin resistance has not been well explained.

Downregulation of miR-186-5p was well documented in NSCLC tumor tissues and cell lines, as well as its anti-tumor role in NSCLC cells both in vitro and in vivo. However, very recently, Feng et al. [37] published a conflicting announcement that miR-186-5p was upregulated in lung ADC tumor tissues and cell lines (H1299 and NCI-H1975) as compared with tumor-adjacent tissues and normal human lung epithelial cells, respectively. Notably, their finding was in consistent with data from Starbase v3.0 and they further observed that this upregulation accelerated lung ADC cell proliferation, migration and invasion through targeting PTEN. Unfortunately, they did not discuss the causes of this controversial outcome comparing to previous studies, as well as the results in this study. It remains a big question that should be concerned and settled in the further.

In conclusion, we showed that miR-186-5p was downregulated in DDP resistant NSCLC tissues and cells. Ectopic expression of miR-186a-5p sensitized DDP resistant NSCLC cells to DDP in vitro and in vivo through targeting SIX1. Our findings supported miR-186/SIX1 axis might be a promising biomarker and target for the treatment and prognosis of DDP resistant NSCLC.

Acknowledgements: This work was supported by the project of National Natural Science Fund (Grant No. 8170110347, the project of Basic and Frontier Research of Chongqing (Grant No. cstc2016jcyA0286), and the inner topics of southwest hospital (Grant No. SWH2016JCYB-56). 


\section{References}

[1] JEMAL A, BRAY F, CENTER MM, FERLAY J, WARD E et al. Global cancer statistics. CA Cancer J Clin 2011; 61: 69-90. https://doi.org/10.3322/caac.20107

[2] EBERHARDT WE, DE RUYSSCHER D, WEDER W, LE PECHOUX C, DE LEYN $P$ et al. 2nd ESMO Consensus Conference in Lung Cancer: locally advanced stage III nonsmall-cell lung cancer. Ann Oncol 2015; 26: 1573-1588. https://doi.org/10.1093/annonc/mdv187

[3] ROSE MC, KOSTYANOVSKAYA E, HUANG RS. Pharmacogenomics of cisplatin sensitivity in non-small cell lung cancer. Genomics Proteomics Bioinformatics 2014; 12: 198209. https://doi.org/10.1016/j.gpb.2014.10.003

[4] CARBONE DP, FELIP E. Adjuvant therapy in non-small cell lung cancer: future treatment prospects and paradigms. Clin Lung Cancer 2011; 12: 261-271. https://doi.org/10.1016/j. cllc.2011.06.002

[5] DONG H, LEI J, DING L, WEN Y, JU H et al. MicroRNA: function, detection, and bioanalysis. Chem Rev 2013; 113: 6207-6233. https://doi.org/10.1021/cr300362f

[6] ORTHOLAN C, PUISSEGUR MP, ILIE M, BARBRY P, MARI B et al. MicroRNAs and lung cancer: new oncogenes and tumor suppressors, new prognostic factors and potential therapeutic targets. Curr Med Chem 2009; 16: 1047-1061. https://doi.org/10.2174/092986709787581833

[7] TAN S, WU Y, ZHANG CY, LI J. Potential microRNA targets for cancer chemotherapy. Curr Med Chem 2013; 20: 3574-3581. https://doi.org/10.2174/0929867311320290003

[8] WANG H, SHEN Q, ZHANG X, YANG C, CUI S et al. The Long Non-Coding RNA XIST Controls Non-Small Cell Lung Cancer Proliferation and Invasion by Modulating miR186-5p. Cell Physiol Biochem 2017; 41: 2221-2229. https:// doi.org/10.1159/000475637

[9] HUANG T, WANG G, YANG L, PENG B, WEN Y et al. MiR186 inhibits proliferation, migration, and invasion of nonsmall cell lung cancer cells by downregulating Yin Yang 1. Cancer Biomark 2017; 21: 221-228. https://doi.org/10.3233/ CBM-170670

[10] CAI J, WU J, ZHANG H, FANG L, HUANG Y et al. miR186 downregulation correlates with poor survival in lung adenocarcinoma, where it interferes with cell-cycle regulation. Cancer Res 2013; 73: 756-766. https://doi.org/10.1158/00085472.CAN-12-2651

[11] YE J, ZHANG Z, SUN L, FANG Y, XU X et al. miR-186 regulates chemo-sensitivity to paclitaxel via targeting MAPT in non-small cell lung cancer (NSCLC). Mol Biosyst 2016; 12: 3417-3424. https://doi.org/10.1039/c6mb00576d

[12] SUN KX, JIAO JW, CHEN S, LIU BL, ZHAO Y. MicroRNA-186 induces sensitivity of ovarian cancer cells to paclitaxel and cisplatin by targeting ABCB1. J Ovarian Res 2015; 8: 80. https://doi.org/10.1186/s13048-015-0207-6

[13] LI C, GAO Y, LI Y, DING D. TUG1 mediates methotrexate resistance in colorectal cancer via miR-186/CPEB2 axis. Biochem Biophys Res Commun 2017; 491: 552-557. https://doi. org/10.1016/j.bbrc.2017.03.042
[14] BROZOVIC A. The relationship between platinum drug resistance and epithelial-mesenchymal transition. Arch Toxicol 2017; 91: 605-619. https://doi.org/10.1007/s00204-0161912-7

[15] LI J, SONG J, GUO F. miR-186 reverses cisplatin resistance and inhibits the formation of the glioblastoma-initiating cell phenotype by degrading Yin Yang 1 in glioblastoma. Int J Mol Med 2019; 43: 517-524. https://doi.org/10.3892/ ijmm.2018.3940

[16] ZHU X, SHEN H, YIN X, LONG L, XIE C et al. miR-186 regulation of Twist1 and ovarian cancer sensitivity to cisplatin. Oncogene 2016; 35: 323-332. https://doi.org/10.1038/ onc. 2015.84

[17] VAN KOUWENHOVE M, KEDDE M, AGAMI R. MicroRNA regulation by RNA-binding proteins and its implications for cancer. Nat Rev Cancer 2011; 11: 644-656. https://doi. org/10.1038/nrc3107

[18] TISEO M, FRANCIOSI V, GROSSI F, ARDIZZONI A. Adjuvant chemotherapy for non-small cell lung cancer: ready for clinical practice? Eur J Cancer 2006; 42: 8-16. https://doi. org/10.1016/j.ejca.2005.08.031

[19] ZHANG J, ZHANG T, TI X, SHI J, WU C et al. Curcumin promotes apoptosis in A549/DDP multidrug-resistant human lung adenocarcinoma cells through an miRNA signaling pathway. Biochem Biophys Res Commun 2010; 399: 1-6. https://doi.org/10.1016/j.bbrc.2010.07.013

[20] BRAY F, FERLAY J, SOERJOMATARAM I, SIEGEL RL, TORRE LA et al. Global cancer statistics 2018: GLOBOCAN estimates of incidence and mortality worldwide for 36 cancers in 185 countries. CA Cancer J Clin 2018; 68: 394-424. https://doi.org/10.3322/caac.21492

[21] FADEJEVA I, OLSCHEWSKI H, HRZENJAK A. MicroRNAs as regulators of cisplatin-resistance in non-small cell lung carcinomas. Oncotarget 2017; 8: 115754-115773. https://doi.org/10.18632/oncotarget.22975

[22] ZANG YS, ZHONG YF, FANG Z, LI B, AN J. MiR-155 inhibits the sensitivity of lung cancer cells to cisplatin via negative regulation of Apaf-1 expression. Cancer Gene Ther 2012; 19: 773-778. https://doi.org/10.1038/cgt.2012.60

[23] WANG H, ZHU LJ, YANG YC, WANG ZX, WANG R. MiR224 promotes the chemoresistance of human lung adenocarcinoma cells to cisplatin via regulating $\mathrm{G}(1) / \mathrm{S}$ transition and apoptosis by targeting p21(WAF1/CIP1). Br J Cancer 2014; 111: 339-354. https://doi.org/10.1038/bjc.2014.157

[24] FARHAN M, MALIK A, ULLAH MF, AFAQ S, FAISAL M et al. Garcinol Sensitizes NSCLC Cells to Standard Therapies by Regulating EMT-Modulating miRNAs. Int J Mol Sci 2019; 20. https://doi.org/10.3390/ijms20040800

[25] SUN W, PING W, TIAN Y, ZOU W, LIU J et al. miR-202 Enhances the Anti-Tumor Effect of Cisplatin on Non-Small Cell Lung Cancer by Targeting the Ras/MAPK Pathway. Cell Physiol Biochem 2018; 51: 2160-2171. https://doi. org/10.1159/000495835

[26] LI H, YIN C, ZHANG B, SUN Y, SHI L et al. PTTG1 promotes migration and invasion of human non-small cell lung cancer cells and is modulated by miR-186. Carcinogenesis 2013; 34: 2145-2155. https://doi.org/10.1093/carcin/bgt158 
[27] HUANG T, SHE K, PENG G, WANG W, HUANG J et al. MicroRNA-186 suppresses cell proliferation and metastasis through targeting MAP3K2 in non-small cell lung cancer. Int J Oncol 2016; 49: 1437-1444. https://doi.org/10.3892/ ijo.2016.3637

[28] CUI G, CUI M, LI Y, LIANG Y, LI W et al. MiR-186 targets ROCK1 to suppress the growth and metastasis of NSCLC cells. Tumour Biol 2014; 35: 8933-8937. https://doi. org/10.1007/s13277-014-2168-6

[29] LIU Q, LI A, TIAN Y, LIU Y, LI T et al. The expression profile and clinic significance of the SIX family in non-small cell lung cancer. J Hematol Oncol 2016; 9: 119. https://doi. org/10.1186/s13045-016-0339-1

[30] GRIER DG, THOMPSON A, KWASNIEWSKA A, MCGONIGLE GJ, HALLIDAY HL et al. The pathophysiology of HOX genes and their role in cancer. J Pathol 2005; 205: 154-171. https://doi.org/10.1002/path.1710

[31] ARMAT M, RAMEZANI F, MOLAVI O, SABZICHI M, SAMADI N. Six family of homeobox genes and related mechanisms in tumorigenesis protocols. Tumori 2016; 2016: 236-243. https://doi.org/10.5301/tj.5000495

[32] MIMAE T, OKADA M, HAGIYAMA M, MIYATA Y, TSUTANI Y et al. Upregulation of notch2 and six1 is associated with progression of early-stage lung adenocarcinoma and a more aggressive phenotype at advanced stages. Clin Cancer Res 2012; 18: 945-955. https://doi.org/10.1158/1078-0432. CCR-11-1946
[33] XIA Y, ZHU Y, MA T, PAN C, WANG J et al. miR-204 functions as a tumor suppressor by regulating SIX1 in NSCLC. FEBS Lett 2014; 588: 3703-3712. https://doi.org/10.1016/j. febslet.2014.08.016

[34] LI Z, TIAN T, HU X, ZHANG X, NAN F et al. Six1 mediates resistance to paclitaxel in breast cancer cells. Biochem Biophys Res Commun 2013; 441: 538-543. https://doi. org/10.1016/j.bbrc.2013.10.131

[35] BEHBAKHT K, QAMAR L, ALDRIDGE CS, COLETTA $\mathrm{RD}$, DAVIDSON SA et al. Six1 overexpression in ovarian carcinoma causes resistance to TRAIL-mediated apoptosis and is associated with poor survival. Cancer Res 2007; 67: 3036-3042. https://doi.org/10.1158/0008-5472.CAN-063755

[36] YANG Z, FENG Z, GU J, LI X, DONG Q et al. microRNA-488 inhibits chemoresistance of ovarian cancer cells by targeting Six 1 and mitochondrial function. Oncotarget 2017; 8: 80981-80993. https://doi.org/10.18632/oncotarget.20941

[37] FENG H, ZHANG Z, QING X, FRENCH SW, LIU D. Mir-186-5p promotes cell growth, migration and invasion of lung adenocarcinoma by targeting pten. Exp Mol Pathol 2019; 108: 105-113. https://doi.org/10.1016/j.yexmp.2019.04.007 